https://doi.org/10.15407/ujpe64.8.705

M.V.T. MACHADO

High Energy Physics Phenomenology Group, GFPAE IF-UFRGS

Caixa Postal 15051, CEP 91501-970

(Av. Bento Goncalves 9500, Porto Alegre, RS, Brazil; e-mail: magnus@if.ufrgs.br)

\title{
INVESTIGATING THE SOFT PROCESSES WITHIN THE QCD COLOR DIPOLE PICTURE
}

We consider the QCD parton saturation models to describe the soft interactions at the highenergy limit. The total and elastic cross-sections, as well as the elastic slope parameter, are obtained for proton-proton and pion-proton collisions and compared to recent experimental results.

Keywords: color dipole picture, QCD parton saturation, Regge theory.

\section{Introduction}

Describing the soft processes with the use of the QCD degrees of freedom is a quite difficult task, since they are dominated by a long distance (nonperturbative) physics. It has been shown that the soft observables as the total and elastic cross-sections depend on the transition region between the high parton density system (saturation domain) and the perturbative QCD region [1-3]. The parton saturation phenomenon [4-6] is a well-established property of highenergy systems and gives a high-quality description of inclusive and exclusive deep inelastic scattering (DIS) data. As evidences of the successfulness of such approach, we quote the description of the light meson photoproduction cross-section [7-12] and diffractive DIS (DDIS) [13, 14]. Both are semihard processes, where an important contribution to the cross-section comes from the kinematic region in a vicinity of the saturation momentum, $Q_{s}$. This dimensional scale increases in the high-energy region. A well-known formalism, which is intuitive, and where the saturation physics can be easily implemented, is the QCD color dipole picture. It is expected [1] that the soft processes measured, for instance, at the Large Hadron Collider (LHC) in hadron-hadron collisions probe the distances about $r \sim 1 / Q_{s} \ll R_{h}$, with $R_{h}$ being the hadron radius. In this context, the hadron scattering at the LHC could be described by color dipoles as the correct degrees of freedom even at large transverse distances. Moreover, it has been shown that the crosssections for soft hadron-hadron collisions within satu-

(C) M.V.T. MACHADO, 2019

ISSN 2071-0194. Ukr. J. Phys. 2019. Vol. 64, No. 8 ration approaches satisfy the Froissart-Martin bound $[2,3]$. In this context, the role played by the unitarized hard Pomeron contribution to the soft observables has been carefully discussed in Refs. [15, 16].

Here, we will investigate the soft observable in the small- $t$ regime within the color dipole picture and parton saturation approaches. The paper is organized as follows. In the next section, we summarize the theoretical information to compute the cross-section for hadron-hadron collisions in two color dipole approaches. First, we consider the asymptotic crosssection following Ref. [3], where the $p p$ cross-section is assumed to be dominated by the two-gluon production in the final state, $p p \rightarrow g g+X$. There, the main ingredients are the gluon distribution of a projectile and the partonic cross-section associates to the interaction $g N \rightarrow g g+X$. We also consider the model presented in Ref. [1], where the virtual photon wave-function is replaced by the corresponding wavefunction for the hadron projectile. The hadron-proton interaction is computed using the dipole-proton amplitude constrained from DIS data. The numerical results from both models are compared to experimental measurements focusing in the LHC kinematic regime. Finally, we discuss the main theoretical uncertainties and present the main conclusions.

\section{Theoretical Frameworks and Their Phenomenological Applications}

Our first investigation will consider the color dipole approach applied to hadron-hadron collisions proposed in Refs. [3]. For simplicity, we address initially the case for proton-proton collisions in colliders. The 
formalism is able to provide us the production crosssection of (heavy or light) quark pairs or gluons at the final state. Namely, similarly to photon-hadron interactions, the total quark production cross-section is given by $[17,18]$

$$
\begin{aligned}
& \sigma(p p \rightarrow q \bar{q} X)=2 \int_{0}^{-\ln \left(\frac{2 m_{q}}{\sqrt{s}}\right)} d y x_{1} G\left(x_{1}, \mu_{F}^{2}\right) \times \\
& \times \sigma(G N \rightarrow q \bar{q} X),
\end{aligned}
$$

where $y=\frac{1}{2} \ln \left(x_{1} / x_{2}\right)$ is the rapidity of the pair, $\mu_{F} \sim m_{Q}$ is the factorization scale. The quantity $x_{1} G\left(x_{1}, \mu_{F}^{2}\right)$ is the projectile gluon density on the scale $\mu_{F}$, and the partonic cross-section $\sigma(G N \rightarrow$ $\rightarrow q \bar{q} X)$ is given by [17]

$\sigma(G N \rightarrow q \bar{q} X)=\int d z d^{2}\left|\Psi_{G \rightarrow q \bar{q}}(z,)\right|^{2} \sigma_{q \bar{q} G}(z)$,

with $\Psi_{G \rightarrow q \bar{q}}$ being the pQCD calculated distribution amplitude, which describes the dependence of the $|q \bar{q}\rangle$ Fock component on the transverse separation and the fractional momentum. It is given by,

$$
\begin{aligned}
& \left|\Psi_{G \rightarrow q \bar{q}}(z, \mathbf{R})\right|^{2}=\frac{\alpha_{s}\left(\mu_{R}\right)}{(2 \pi)^{2}}\left\{m_{q}^{2} k_{0}^{2}\left(m_{q} r\right)+\right. \\
& \left.+\left[z^{2}+(1-z)^{2}\right] m_{q}^{2} k_{1}^{2}\left(m_{q} r\right)\right\},
\end{aligned}
$$

where $\alpha_{s}\left(\mu_{R}\right)$ is the strong coupling constant, which is probed on a renormalization scale $\mu_{R} \sim m_{Q}$. We note that the wavefunction will lead to a dominance of dipole sizes around $r \sim 1 / m_{q}$ in the corresponding $r$-integration. Therefore, for the heavy quark production, the color transparency behavior from the dipole cross-section, $\sigma_{\text {dip }}(r) \propto r^{2}$, will be the main contribution (pQCD). In the charm case, an important contribution should come from the saturation region, since the typical dipole size, $r \simeq 1 \mathrm{GeV}^{-1}$, can reach an order of magnitude similar to the saturation radius, $R_{s}(x)=1 / Q_{s}(x) \propto(\sqrt{s})^{-\lambda / 2}$ (with $\lambda \simeq$ $\simeq 0.3$. On the other hand, for light quarks, $m_{q} \simeq$ $\simeq 0.14 \mathrm{GeV}$, we are deep in the parton saturation (very low- $x_{2}$ and a small scale of the probe) and nonperturbative regions. This will be the case in the following calculation.

In the partonic cross-section, $\sigma_{q \bar{q} G}$ is the crosssection for the scattering of a color neutral quarkantiquark-gluon system on the target and is directly connected with the dipole cross-section:

$\sigma_{q \bar{q} G}=\frac{9}{8}\left[\sigma_{\text {dip }}\left(x_{2}, z \mathbf{R}\right)+\sigma_{\text {dip }}\left(x_{2}, \bar{z} \mathbf{R}\right)\right]-$ $-\frac{1}{8} \sigma_{\operatorname{dip}}\left(x_{2}, \mathbf{R}\right)$

Here, the main idea is that, at high energies, a gluon $G$ from the projectile hadron can develop a fluctuation which contains a $Q \bar{Q}$ pair. Interaction with the color field of a target then may release these heavy quarks. Such an approach is valid for high energies, where the coherence length $l_{c} \approx 1 / x_{2}$ is larger than the target radius. Therefore, it is natural to include the parton saturation effects and to use the fact the dipole cross-section is universal, i.e., it is process-independent. For the sake of completeness, the parton momentum fractions are written in terms of the quark pair rapidity and masses, $x_{1,2}=\frac{2 m_{Q}}{\sqrt{s}} \exp ( \pm y)$.

Following Ref. [3], we obtain the asymptotic hadron-hadron cross-section within the color dipole approach considering the dominant process, $p p \rightarrow$ $\rightarrow G G X$, at high energies. Now, the gluon $G$ from the projectile hadron develops a fluctuation which contains a two-gluon $(G G)$ pair further interacting with target's color field. Accordingly, the expression for the total cross-section for the gluon production at the final state is given by [19],

$$
\begin{aligned}
& \sigma(p p \rightarrow G G X)=2 \int_{0}^{-\ln \left(\frac{2 m_{G}}{\sqrt{s}}\right)} d y x_{1} G\left(x_{1}, \mu_{F}^{2}\right) \times \\
& \times \sigma(G N \rightarrow G G X),
\end{aligned}
$$

where the effective gluon mass, $m_{G}$, was introduced in order to regularize the calculation. Thus, in this case, one has $x_{1,2}=\frac{2 m_{G}}{\sqrt{s}} \exp ( \pm y)$.

The new partonic cross-section $\sigma(G N \rightarrow G G X)$ is given by

$\sigma(G N \rightarrow G G X)=$

$=\int d z d^{2} \mathbf{R}\left|\Psi_{G \rightarrow G G}(z, \mathbf{R})\right|^{2} \sigma_{G G G}(z, \mathbf{R})$

with $\Psi_{G \rightarrow G G}$ being the corresponding distribution amplitude associated with the $|G G\rangle$ Fock state. It is obtained from Eq. (2) in the following way: $\left|\Psi_{G \rightarrow G G}\right|^{2}=2\left(N_{c}-1\right)\left|\Psi_{G \rightarrow q \bar{q}}\right|^{2}$. The partonic crosssection $\sigma_{G G G}$ is the cross-section for the scattering a a color neutral three-gluon system on the target and

ISSN 2071-0194. Ukr. J. Phys. 2019. Vol. 64, No. 8 
is directly related to the dipole cross-section in the following way [19]:

$\sigma_{G G G}=\frac{1}{2}\left[\sigma_{\mathrm{dip}}\left(x_{2}, z \mathbf{R}\right)+\sigma_{\mathrm{dip}}\left(x_{2}, \bar{z} \mathbf{R}\right)+\sigma_{\mathrm{dip}}\left(x_{2}, \mathbf{R}\right)\right]$

Now, we will present the corresponding phenomenology using Eq. (4). From Ref. [3], we identify basically two main shortcomings: a very low value for the effective gluon mass, $m_{G}=154 \mathrm{MeV}<\Lambda_{Q C D}$ and the identification of the scale $\mu$ with the starting evolution scale in the gluon PDFs considered, $\mu^{2}=$ $=Q_{0}^{2}$. Here, we will use the value $m_{G}=400 \mathrm{MeV}$. Moreover, for the gluon PDF probed on the low scale, $\mu^{2}=m_{G}^{2}=0.16 \mathrm{GeV}^{2}$ will be given for a prediction from the parton saturation physics,

$x G\left(x, Q^{2}\right)=\frac{3 \sigma_{0} Q_{s}^{2}}{4 \pi^{2} \alpha_{s}}\left[1-\left(1+\frac{Q^{2}}{Q_{s}^{2}}\right) e^{-\frac{Q^{2}}{Q_{s}^{2}}}\right]$,

where the updated values for the GBW model parameters have been used [20]. Consistently, for the dipole cross-section, we have used the GBW parametrization. It should be noted that the result is parameterfree and corresponds to the soft Pomeron contribution to the cross-section.

In Fig. 1, the result for the total cross-section in proton-proton collisions is presented. Both the lowenergy and cosmic rays data are presented. Experimental measurements from colliders are properly identified [21], especially the recent LHC data. The asymptotic model is in a quite good agreement compared to accelerator data, despite no further adjustment has been done. There is some room for fitting the reggeon contribution at low energies.

We have also considered another color dipole approach addressing the soft scattering processes. In such a case, other observables can be described as the elastic cross-section and the elastic slope parameter. We follow Ref. [1] and compute the total crosssection in following way:

$$
\begin{aligned}
& \sigma_{\text {tot }}^{h p}(\sqrt{s})= \\
& =2 \int d^{2} b \int_{0}^{1} d z \int d^{2} r\left|\Psi_{h}(r, z)\right|^{2} N(s, r, b) .
\end{aligned}
$$

It depends on the color dipole amplitude, $N(s, r, b)$, and on the hadron wavefunction, $\Psi_{h}(r, z)$. The expressions resembles the same equation for the DIS

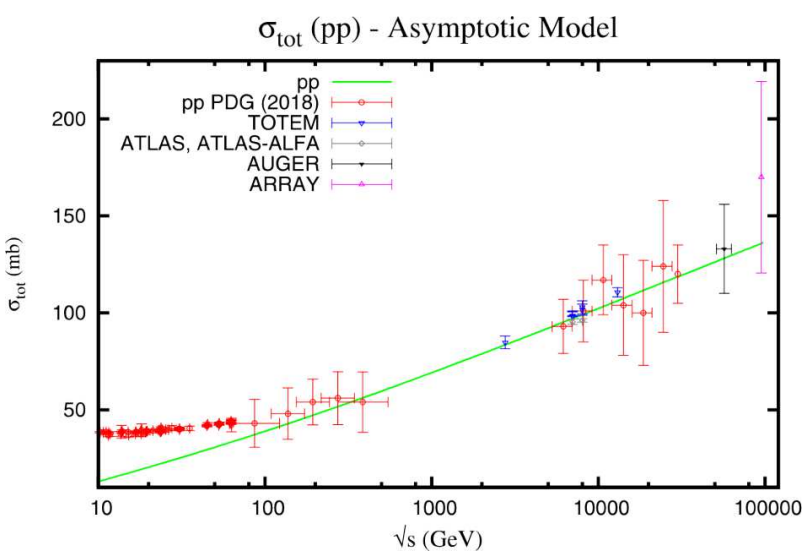

Fig. 1. ( $p p$ total cross-section as a function of the center-ofmass-energy, including low-energy and cosmic rays data. The LHC data are explicitly identified (TOTEM and ATLAS Collaborations)

description within the color dipole approach. In other words, the photon wavefunction is replaced by the hadron one. Here, in the meson-proton scattering, a meson is treated as a $q \bar{q}$ pair: the calculation implies that DIS, i.e., the interaction of a color dipole with a proton target and the saturation physics can be embedded in the dipole amplitude. A similar approach has been considered also in Refs. [22-24], where the Pomeron dynamics is written in terms of the dipoledipole cross-section. For instance, in Ref. [22], the large dipoles are dominated by a soft Pomeron contribution, whereas small dipoles are driven by a hard Pomeron piece (two-Pomeron model with hard and soft Pomerons). On the other hand, in Ref. [23, 24] based on Mueller's cascade model, the authors discussed several contributions including the effect of Pomeron loops.

To characterize mesons and baryons, we use the phenomenological ansatz from Wirbel-Stech-Bauer (WSB) [22] which gives

$\psi_{h}(z, \mathbf{r})=\sqrt{\frac{z(1-z)}{2 \pi S_{h}^{2} N_{h}}} e^{-\left(z-\frac{1}{2}\right)^{2} /\left(4 \Delta z_{h}^{2}\right)} e^{-|\mathbf{r}|^{2} /\left(4 S_{h}^{2}\right)}$,

where the hadron wave function normalization to unity, $\int d z d^{2} r\left|\psi_{h}(z, \mathbf{r})\right|^{2}=1$, requires the following normalization constant:

$N_{h}=\int_{0}^{1} d z z(1-z) e^{-\left(z-\frac{1}{2}\right)^{2} /\left(2 \Delta z_{h}^{2}\right)}$. 


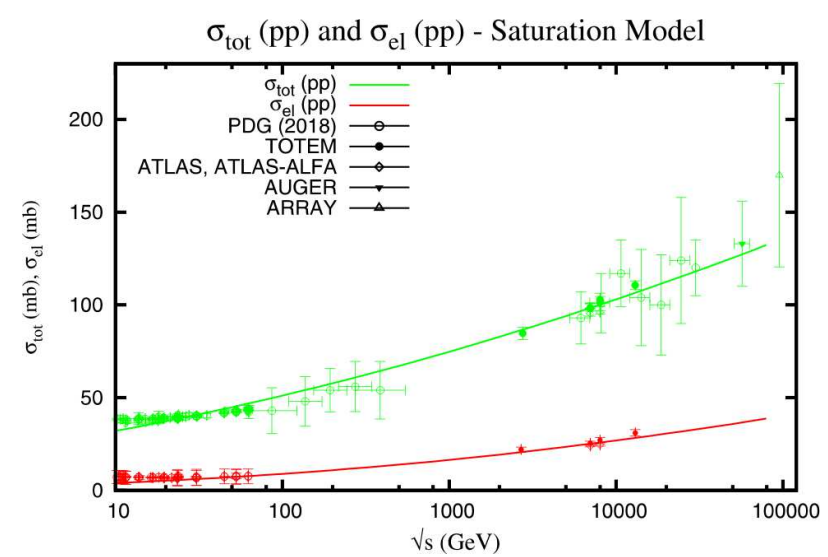

Fig. 2. The total and elastic cross-sections for $p p$ collisions The upper cross-sections are total cross-sections, while the lower ones are the elastic cross-sections. Tevatron, SPS, LHC, and cosmic rays data are presented [21]. The lines are the results from the eikonal-type (saturation) model

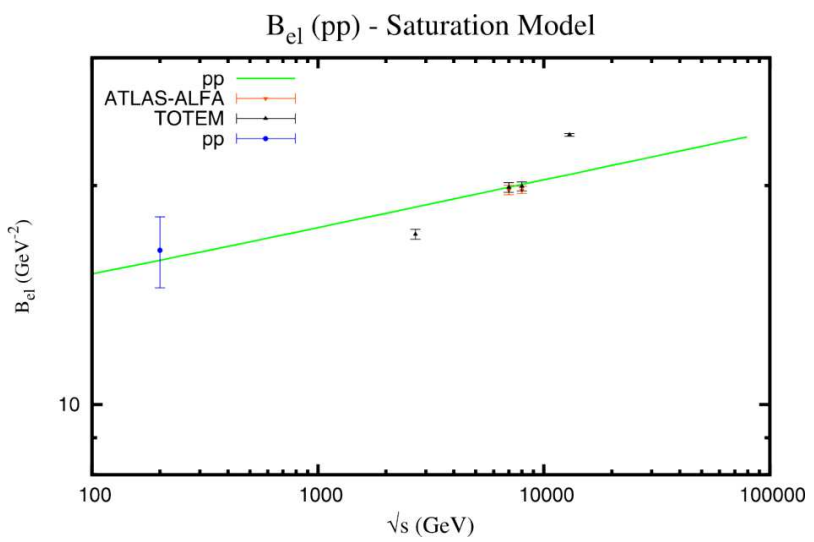

Fig. 3. Slope $B_{\mathrm{el}}(s)$ for the $p p$ elastic scattering as a function of $\sqrt{s}$. The recent LHC data from TOTEM and ATLAS-ALFA are presented

Therefore, mesons and baryons are assumed to have a $q \bar{q}$ and quark-diquark valence structure. Since quark-diquark systems are equivalent to $q \bar{q}$ systems, this allows us to model not only mesons but also baryons as color-dipoles. The values of the parameters in our case are the following: $\Delta z_{h}=0.3(2)$ and $S_{h}=0.86(0.607) \mathrm{fm}$, for $p / \bar{p}\left(\pi^{ \pm}\right)$, respectively $[22]$.

Before discussing an impact-parameter dipole amplitude extracted from DIS data, we would need to rewrite the energy dependence from the photonhadron scattering in terms of the appropriate Bjorken scaling variable- $x$. In this work, the following ansatz has been considered:

$\frac{1}{x}=\frac{s r^{2}}{\left(s_{0} R_{c}^{2}\right)}$,

which has been successfully considered in Ref. [25]. Here, $s_{0}^{2} \sim m_{h}^{2}$ and $R_{c}=0.2 \mathrm{fm}$. Such an ansatz is numerically equivalent to the proposal $\frac{1}{x}=\frac{s}{Q_{0}^{2}}$, with $Q_{0}^{2} \sim\left(2 m_{q}\right)^{2} \simeq m_{h}^{2}$, done in Ref. [1]. For simplicity and faster numerical calculations, we consider the last relation, where the $Q_{0}^{2}$ parameter will be extracted from the total cross-section data.

We tested an eikonal-like expression for the dipole amplitude, where the impact parameter dependence is factorized from the energy dependence. The function $S(b)$ is described by the dipole profile function. Namely, the amplitude has the following form:

$$
\begin{aligned}
& N(x, r, b)=1-\exp \left(-\frac{1}{2} \hat{\sigma}(x, r) S(b)\right), \\
& \hat{\sigma}(x, r)=\sigma_{0} \frac{\left(r Q_{s}(x)\right)^{2}}{4}, \quad S(b)=\frac{2 \beta b}{\pi R^{2}} K_{1}(\beta b),
\end{aligned}
$$

where we have considered the parameters for $\hat{\sigma}$ from the GBW saturation model [20] and the value $R^{2}=$ $=4.5 \mathrm{GeV}^{-2}$. Here, the parameter $\beta$ was defined as $\beta=\frac{\sqrt{8}}{R}$. In Fig. 2, we present the results associated with the application of the model for the $b$-dependent color dipole amplitude to the $p p$ scattering at the accelerator energy regime. Accordingly, we can say that the model adequately describes the proton-proton cross-section data, and we extend it to higher energies to make predictions for cosmic-ray energies. Moreover, in Fig. 3, we present the slope parameter, $B_{\mathrm{el}}(s)$ as a function of the center-of-mass energy. We present the comparison against the recent LHC data, and it was found that the description of data is quite reasonable.

In summary, we have applied the color dipole picture to the soft hadron-hadron scattering, by including the parton saturation phenomenon as the transition region between the soft and hard domains. We have shown that the inclusive process is mainly driven for dipole sizes near the saturation radius in the highenergy regime. The main advantage is that the corresponding phenomenology is almost free of parameters, as they are completely constrained from DIS data in $e p$ interactions. The models rely on the dipole cross-section or $b$-dependent dipole amplitude and indicate that the impact parameter profile is crucial for a good data description. The advent of the 
LHC opened a new window for the studies of the diffraction and the elastic and inelastic scatterings, as they are not strongly contaminated by non-diffractive events. This is translated in the Regge-theory language saying that the scattering amplitude is completely determined by a Pomeron exchange. The current measurements on these soft observables at the LHC in proton-proton collisions are in a very good shape, covering the energies of $0.9,2.76,7,8$, and $13 \mathrm{TeV}[21]$. In the context of the saturation physics, the soft Pomeron may be understood as an unitarized perturbation Pomeron [26]. It can be shown that the trajectory of a soft Pomeron could emerge as a result of the interplay between perturbative physics of a hard Pormeron and the confining properties of the QCD vacuum. Specifically, the local unitarization in the impact parameter plane can lead to a reasonable description of the intercept and the slope of a soft Pomeron [26]. Our work corroborates those statements, once the soft observable in the small- $t$ regime is correctly described within the color dipole picture and the parton saturation approach.

The author thanks the conference organizers and other presenters for making this valuable opportunity available to all of us. Thank you for your gracious hospitality and professionalism. This work was partially financed by the Brazilian funding agencies $C N P q$ and $C A P E S$.

1. J. Bartels, E. Gotsman, E. Levin, M. Lublinsky, U. Maor. The Dipole picture and saturation in soft processes. Phys. Lett. B 556, 114 (2003).

2. F. Carvalho, F. O. Duraes, V. P. Goncalves, F.S. Navarra. Gluon saturation and the Froissart bound: A Simple approach. Mod. Phys. Lett. A 23, 2847 (2008).

3. C.A. Argueelles, F. Halzen, L. Wille, M. Kroll, M.H. Reno. High-energy behavior of photon, neutrino, and proton cross sections. Phys. Rev. D 92, 074040 (2015).

4. F. Gelis, E. Iancu, J. Jalilian-Marian, R. Venugopalan. Ann. Rev. Nucl. Part. Sci. 60, 463 (2010).

5. H. Weigert. Evolution at small $x_{b} j$ : The color glass condensate. Prog. Part. Nucl. Phys. 55, 461 (2005).

6. J. Jalilian-Marian, Y.V. Kovchegov. Saturation physics and deuteron-gold collisions at RHIC Prog. Part. Nucl. Phys. 56, 104 (2006).

7. A.C. Caldwell, M.S. Soares. Vector meson production in the Golec-Biernat Wuesthoff model. Nucl. Phys. A 696, 125 (2001);

8. H. Kowalski, D. Teaney. An impact parameter dipole saturation model. Phys. Rev. D 68, 114005 (2003).
9. J.R. Forshaw, R. Sandapen, G. Shaw. Color dipoles and $\rho, \varphi$ electroproduction. Phys. Rev. D 69, 094013 (2004).

10. C. Marquet, R. Peschanski, G. Soyez. Exclusive vector meson production at HERA from QCD with saturation. Phys. Rev. D 76, 034011 (2007).

11. H. Kowalski, L. Motyka, G. Watt. Exclusive diffractive processes at HERA within the dipole picture. Phys. Rev. D 74, 074016 (2006).

12. N. Armesto, A.H. Rezaeain. Phys. Rev. D 90, 054003 (2014).

13. J.R. Forshaw, R. Sandapen, G. Shaw. Further success of the colour dipole model. JHEP 0611, 025 (2006).

14. K. Golec-Biernat, M.Wüsthoff. Saturation in diffractive deep inelastic scattering. Phys. Rev. D 60, 114023 (1999).

15. J.-R. Cudell, O. V. Selyugin. Unitarisation and black-disk limit at the LHC in the presence of a hard pomeron. Phys. Lett. B 662, 417 (2008).

16. J.-R. Cudell, E. Predazzi, O.V. Selyugin. New analytic unitarisation schemes. Phys. Rev. D 79, 034033 (2009).

17. N.N. Nikolaev, G. Piller, B.G. Zakharov. Inclusive heavyflavor production from nuclei. Z. Phys. A 354, 99 (1996).

18. B.Z. Kopeliovich, A.V. Tarasov. Gluon shadowing in heavy flavor production off nuclei. Nucl. Phys. A 710, 180 (2002).

19. N.N. Nikolaev, W. Schafer, B.G. Zakharov. Nonlinear $k \perp$ factorization for gluon-gluon dijets produced off nuclear targets Phys. Rev. D 72, 114018 (2005).

20. K. Golec-Biernat, S. Sapeta. Saturation model of DIS: An update. JHEP 1803, 102 (2018).

21. M. Tanabashi et al., (Particle Data Group). Review of Particle Physics. Phys. Rev. D 98, 030001 (2018).

22. A.I. Shoshi, F.D. Steffen, H.J. Pirner. S-matrix unitarity, impact parameter profiles, gluon saturation and highenergy scattering. Nucl. Phys. A 709, 131 (2002).

23. C. Flensburg, G. Gustafson, L. Lonnblad. Elastic and quasi-elastic pp and $y^{*} p$ scattering in the dipole model. Eur. Phys. J. C 60, 233 (2009)

24. G. Gustafson, L. Lonnblad, A. Ster, T. Csorgo. Total, inelastic and (quasi-)elastic cross sections of high energy pA and $\gamma^{*}$ A reactions with the dipole formalism. JHEP 1510, 022 (2015).

25. A. Donnachie, H.G. Dosch. Comprehensive approach to structure functions. Phys. Rev. D 65, 014019 (2002).

26. L. Motyka. Soft pomeron trajectory from a saturation model. Acta Phys. Polon. B 34, 3069 (2003).

Received 08.07.19

\section{M.B.T. Машадо}

\section{ДОСЛІДЖЕННЯ М'ЯКИХ}

ПРОЦЕСІВ В РАМКАХ КОЛЬОРОВОГО

ДИПОЛЬНОГО ПІДХОДУ КХД

$\mathrm{P}$ е $з$ ю м е

В роботі ми розглядаємо КХД-партонну модель насичення для опису м'яких процесів при високих енергіях. Отримано повний та пружний перерізи, а також параметр нахилу для розсіяння протонів на протонах та піонів на протонах. Ці результати порівнюються з експерименальними даними. 